\title{
Aarnoo Análise das coberturas vacinais de crianças menores de um ano em Roraima, 2013-2017*
}

doi: 10.1590/S1679-49742021000200010

\author{
Analysis of vaccination coverage of children under one year old in Roraima, Brazil, 2013-2017 \\ Analizar la cobertura de vacunación de los menores de un año en Roraima, Brasil, 2013 y 2017
}

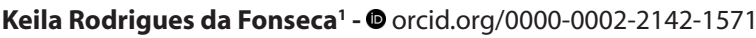

Sandra Maria Franco Buenafuente' - (D orcid.org/0000-0003-2549-3596

Universidade Federal de Roraima, Programa de Pós-Graduação em Ciências da Saúde, Boa Vista, RR, Brasil

\section{Resumo}

Objetivo: Analisar as coberturas vacinais de crianças menores de 1 ano em Roraima, Brasil, entre 2013 e 2017, e expor as percepções dos profissionais de saúde quanto às barreiras que influenciaram no alcance de elevadas coberturas vacinais do estado em 2017. Métodos: Estudo descritivo que analisou as coberturas vacinais para bacilo de Calmette e Guérin (BCG), rotavírus, poliomielite, febre amarela, pentavalente, meningocócica conjugada $\mathrm{C}$ e pneumocócica 10-valente, baseado em dados dos sistemas de informações de imunizações. As barreiras percebidas pelos profissionais foram mensuradas por questionário com respostas em escala de Likert. Resultados: A maior cobertura foi da BCG $(146,1 \%)$ em 2014; e a mais baixa, da vacina contra o rotavírus $(70,4 \%)$ em 2013. A principal barreira (56/100) identificada pelos profissionais atuantes no programa (100 respondentes) para melhores coberturas foi a dificuldade de acesso à internet. Conclusão: As baixas coberturas vacinais refletem a influência das barreiras de acesso a vacinação.

Palavras-chave: Cobertura Vacinal; Programas de Imunização; Vacinação; Acesso aos Serviços de Saúde; Epidemiologia Descritiva.

\footnotetext{
*Artigo derivado da dissertação de mestrado intitulada 'Análise situacional do programa de imunizações no estado de Roraima: cumprimento do calendário básico de vacinação no período de 2013 a 2017', defendida por Keila Rodrigues da Fonseca junto ao Programa de Pós-Graduação em Ciências da Saúde da Universidade Federal de Roraima em 2019.
}

Endereço para correspondência:

Keila Rodrigues da Fonseca - Rua Belém, n 144, apto 11, Bairro Nossa Senhora das Graças, Manaus, AM, Brasil. CEP:69053-380

E-mail: enfkeilafonseca@gmail.com 


\section{Introdução}

A vacinação é uma das ações mais seguras e efetivas na eliminação e controle de doenças transmissíveis, além de ser determinante na redução da morbimortalidade, especialmente em crianças, contingente dos mais vulneráveis. ${ }^{1}$ A tecnologia da imunização possibilitou a erradicação de doenças graves, como a varíola em $1980{ }^{2}$

No Brasil, a vacinação é um direito do cidadão. 0 Programa Nacional de Imunizações (PNI), responsável por organizar e coordenar todas as ações de vacinação, busca garantir vacinação para todos os indivíduos, independentemente da classe social e localidade de residência, área rural ou urbana. ${ }^{3} 0$ PNI tem como missão o controle, a erradicação e a eliminação das doenças imunopreveníveis e, para tanto, o Ministério da Saúde estabelece alguns indicadores que subsidiam a análise do programa: cobertura vacinal; taxa de abandono; e taxa de homogeneidade. ${ }^{4}$

\section{A extensa área rural e indígena, além dos migrantes venezuelanos que buscam abrigo no país, são características que podem influenciar o desempenho do PNI nos estados da região Norte.}

Cobertura vacinal consiste na proporção da população-alvo que recebeu o esquema completo da vacina. Por meio desse indicador, é verificada a vulnerabilidade dos sujeitos e os riscos de adquirirem doenças imunopreveníveis. A missão do PNI somente será cumprida com o alcance de coberturas vacinais adequadas. ${ }^{5} \mathrm{~A}$ taxa de abandono exprime a aceitação da imunização pela população e, enquanto um indicador do Programa, aplica-se a vacinas com esquemas multidoses. A taxa de homogeneidade, por sua vez, estima a proporção de municípios com coberturas vacinais adequadas. ${ }^{6}$

Nas últimas décadas, o PNI tem incorporado novas tecnologias de informação, como o Sistema de Informações do Programa Nacional de Imunizações (SI-PNI), além de ter ampliado o calendário vacinal dos brasileiros. Atualmente, o calendário nacional de vacinação infantil contempla 14 imunobiológicos oito deles, vacinas para crianças menores de 1 ano, destinadas a prevenir cerca de 12 doenças. $^{7}$
A crescente complexidade do PNI tem trazido desafios, entre os quais (i) a manutenção de elevadas e homogêneas coberturas vacinais, (ii) o planejamento da logística de produção e distribuição de imunobiológicos, (iii) a identificação de eventos adversos pós-vacinação, (iv) a busca constante pela melhoria da informação, (v) a prevenção da reemergência de doenças já controladas e (vi) a capacitação e atualização de recursos humanos. ${ }^{8}$ Diante de tal complexidade, são recomendadas análises constantes dos serviços de saúde para que estes alcancem alto nível de desempenho nos procedimentos de imunização. ${ }^{9,10}$

No Brasil, a extensa área rural e indígena, além dos migrantes venezuelanos que buscam abrigo no país, são características que podem influenciar 0 desempenho do PNI nos estados da região Norte, ${ }^{4}$ especialmente Roraima, onde o grande desafio constitui integrar, acomodar e promover ações de vacinação para os milhares de cidadãos brasileiros e migrantes do país vizinho, e assim manter toda a sua população protegida contra as doenças imunopreveníves, reduzindo sua vulnerabilidade. ${ }^{11}$

As dificuldades vivenciadas na prática profissional em saúde, diante da reemergência de doenças imunopreveníveis no estado de Roraima, motivaram a realização deste estudo, cujo objetivo foi, primeiramente, analisar as coberturas vacinais de crianças menores de 1 ano em Roraima, entre 2013 e 2017, ademais de colher as percepções dos profissionais de saúde e gestores atuantes no programa de imunizações quanto às barreiras que influenciam 0 alcance de elevadas coberturas vacinais no estado em 2017.

\section{Métodos}

Trata-se de estudo descritivo, realizado em todos os 15 municípios do estado de Roraima, referente ao período de 2013 a 2017.

Segundo dados do Instituto Brasileiro de Geografia e Estatística, a população estimada de Roraima, em 2017, era de 522.636 habitantes, divididos em 15 municípios. ${ }^{12}$

De acordo com os dados do Sistema de Informações Sobre Nascidos Vivos (Sinasc), no período entre 2013 e 2017, nasceram 56.459 crianças vivas no estado. ${ }^{13}$

Foram consultados dados de coberturas vacinais, doses de vacinas administradas e informações sobre a percepção dos profissionais de saúde e gestores do programa de imunizações de Roraima. 
Para análise das coberturas vacinais e doses de vacinas administradas, foram utilizados dados do Sistema de Informações de Avaliação do Programa de Imunizações e do SI-PNI referentes a doses de vacina aplicadas em todas as crianças menores de 1 ano de idade, nascidas vivas entre 2013 e 2015.

Para análise da percepção dos profissionais de saúde, foram considerados elegíveis todos os profissionais e gestores registrados como atuantes no programa de imunizações durante os meses julho e agosto de 2018.

Os dados para análise das coberturas vacinais foram obtidos do SI-PNI e do Sistema de Informações de Avaliação do Programa de Imunizações. Os imunobiológicos escolhidos para o estudo foram a vacina do bacilo de Calmette-Guérin (BCG), as vacinas contra 0 rotavírus humano, a poliomielite e a febre amarela, a pentavalente (DTP/HB/Hib), a meningocócica conjugada $\mathrm{C}$ e a pneumocócica 10 -valente. As variáveis da pesquisa compreenderam os seguintes indicadores de saúde do PNI: cobertura vacinal, taxa de homogeneidade e taxa de abandono.

As coberturas vacinais foram calculadas considerando-se, como numerador, o número de doses aplicadas de determinada vacina, e como denominador, a estimativa de sua população-alvo, multiplicando-se a razão por 100. No grupo populacional analisado, foram consideradas adequadas as coberturas vacinais que atingiram valores iguais ou superiores a: $90 \%$ para BCG e rotavírus humano; $95 \%$ para as vacinas da poliomielite, pentavalente, meningocócica conjugada C e pneumocócica 10 -valente; e $100 \%$ para a vacina da febre amarela.

As taxas de homogeneidade foram calculadas considerando-se, como numerador, o número de municípios com coberturas adequadas para determinada vacina, e como denominador, o número total de municípios do estado, multiplicando-se a razão por 100. Considerou-se uma taxa de homegeneidade adequada se pelo menos $70 \%$ dos municípios atingissem as metas de vacinação preconizadas. ${ }^{13}$

As taxas de abandono (aplicadas às vacinas-multidose em crianças menores de 1 ano) foram calculadas dividindo-se a diferença entre o número de primeiras doses e o número de últimas doses administradas no esquema vacinal, pelo total de primeiras doses, multiplicando-se o resultado da divisão por 100. Foram consideradas taxas de abandono baixas $(<5 \%)$, médias $(\geq 5 \%$ e $<10 \%)$ e altas $(\geq 10 \%) .{ }^{14}$
A percepção dos profissionais e gestores atuantes no programa de imunizações contou com a elaboração e apresentação aos participantes de um instrumento na forma de questionário semiestruturado, baseado em escala de Likert, contemplando aspectos relacionados às fragilidades do processo de implantação do SI-PNI e às dificuldades da população em aderir ao programa. Para análise de possíveis barreiras de acesso às salas de vacinas, o questionário tomou como referência o modelo teórico proposto por Ferreira et al., ${ }^{15}$ estabelecido sob o arco de dimensões e critérios que influenciam o acesso da população à sala de vacina, classificado da seguinte forma: acesso organizacional; acesso geográfico; acesso sociocultural; e acesso econômico.

Com a finalidade de validar o questionário antes de aplicá-lo na população de interesse, foram realizados os seguintes procedimentos: definição de sua estrutura, objetivos e modelo teórico; validação do conteúdo; e por fim, realização de pré-teste com dez pessoas com as mesmas características da população-alvo. Os participantes podiam se abster de responder a qualquer questão, reservando-se-lhes espaço no questionário para a inclusão de outras variáveis que considerassem relevantes.

Quanto às fragilidades no processo de implantação do SI-PNI, o questionário abrangia as seguintes variáveis: falta de qualificação dos profissionais; computadores e sua instalação; dificuldade de acesso à internet; sobrecarga de trabalho; demora no atendimento; e quantidade insuficiente de profissionais. Os profissionais respondiam segundo sua percepção de cada um desses itens, valorada em quatro pontos: 1 - Pouco frágil; 2 - Frágil; 3 -Sem fragilidade; 4 - Muito frágil.

Quanto à percepção dos profissionais sobre as dificuldades da população para aderir ao programa de imunizações, foram consideradas as seguintes variáveis: dificuldade de acesso ao serviço/extensa área rural; hesitação à vacinação; falta de investimento no programa; falta de prioridade para o programa; e falta de recursos financeiros. Os profissionais respondiam segundo sua percepção de cada um desses itens, valorada em quatro pontos: 1 - Nenhuma; 2 - Pouca; 3 - Nem pouca, nem muita; 4 - Muita.

o questionário foi enviado ao endereço eletrônico cadastrado na Secretaria de Estado da Saúde de Roraima por todos os profissionais e gestores registrados como atuantes no programa durante os meses de julho e agosto do ano de 2018. Nos municípios localizados 
até $134 \mathrm{~km}$ da capital Boa Vista, foi realizada visita para coleta de dados e aplicação do questionário presencialmente.

Os dados das coberturas vacinais, taxas de abandono e de homogeneidade em Roraima, coletados dos sistemas de informações, foram tabulados e traduzidos pela metodologia estatística descritiva, e organizados na forma de gráficos e tabelas. As informações coletadas sobre a percepção dos profissionais, a partir de suas respostas ao questionário, foram sistematizadas em planilhas eletrônicas, $\log _{0}$ interpretadas e analisadas frente à teoria fundamentada.

Para identificar a quantidade desejável de amostra, aplicou-se o método de amostragem simples, calculado - para população finita - pela seguinte fórmula:

$$
\mathrm{n}=\mathrm{N} \cdot \mathrm{p} \cdot \mathrm{q} \cdot\left(\mathrm{Z}_{\mathrm{\alpha} / 2)^{2}}\right)^{2} /(\mathrm{N}-1) \cdot(\mathrm{E})^{2}+\mathrm{p} \cdot \mathrm{q} \cdot\left(\mathrm{Z}_{\mathrm{\alpha} / 2}\right)^{2}
$$

Onde:

$\mathrm{n}=$ tamanho da amostra

$\mathrm{N}$ = tamanho da população

$\mathrm{p}=$ proporção de resultados favoráveis da variável na população

$q$ = proporção de resultados desfavoráveis na população

$\mathrm{Z}_{\alpha / 2 \mid}=$ valor crítico para o grau de confiança desejado

$\mathrm{E}=$ erro-padrão da proporção de casos

Desse modo, obteve-se o valor de 184 e nível de significância de 0,05 .

O projeto do estudo foi aprovado pelo Comitê de Ética em Pesquisa da Universidade Federal de Roraima (CEP/ UFRR) em seu Parecer $n^{0}$ 85816518.8.0000.5302, emitido em 5 de abril de 2018. 0 acesso aos sistemas de informações do programa de imunizações foi autorizado pela Coordenação-Geral de Vigilância em Saúde do Estado de Roraima. Os profissionais que responderam ao questionário assinaram o Termo de Consentimento Livre e Esclarecido.

\section{Resultados}

Foi possível coletar dados secundários dos 15 municípios de Roraima, referentes às sete vacinas de interesse. Os questionários foram enviados para 170 endereços de $\boldsymbol{e}$-mail cadastrados no sistema, e recebidas 70 respostas. Outros 60 profissionais foram convidados a responder ao questionário presencialmente, e 30 deles aceitaram conceder as entrevistas. Assim, dos 230 profissionais convidados a participar, 100 assentiram.

A cobertura vacinal da BCG apresentou-se alta durante todo o período estudado, enquanto as metas de cobertura da vacina do rotavírus humano foram atingidas somente nos anos de 2015 (94,2\%) e 2017 $(90,9 \%)$. A vacina da poliomielite alcançou a cobertura esperada somente em 2015 (106,6\%), enquanto a vacina da febre amarela não atingiu a meta de cobertura vacinal preconizada pelo Ministério da Saúde em nenhum ano do período estudado (Tabela 1).

A maior taxa de homogeneidade alcançada correspondeu à vacina pneumocócica 10 -valente no ano de 2016 (80\%). Já as menores taxas de homogeneidade das coberturas vacinais são referentes à vacina BCG, durante todo o período analisado (Figura 1).

As maiores taxas de abandono ocorreram com a vacina da poliomielite em $2013(21,8 \%)$ e 2017 $(30,2 \%)$, seguidas das taxas de abandono da vacina do rotavírus humano em 2013 (18,6\%). As menores taxas de abandono registradas foram para a vacina meningocócica conjugada C (3,2\%) em 2013, pneumocócica 10 -valente $(5,5 \%)$ em 2013 e $(5,3 \%)$ em 2016, e a vacina da poliomielite (5,5\%) em 2015; considerando-se o conjunto do período 2013-2017, a vacina meningocócica conjugada $\mathrm{C}$ referiu o menor somatório de taxa de abandono do programa de vacinações do estado (Figura 2).

A percepção dos profissionais de saúde e gestores relacionadas às especificidades do programa de imunizações do estado de Roraima foi questionada em todos os municípios do estado. 0 percentual de não resposta, considerando-se a amostra prevista, foi de $45,7 \%$. Do total de 100 profissionais e gestores que responderam ao questionário, 39 eram enfermeiros, 44 vacinadores e 17 gestores cujo trabalho com imunizações fazia parte de suas atividades diárias. Os 100 participantes da pesquisa responderam à totalidade das questões propostas.

Foram registrados os valores atribuídos pelos participantes-profissionais de saúde e gestores do programa de imunizações de Roraima para os fatores responsáveis pela situação vacinal do estado, de acordo com sua percepção (Tabela 2). A primeira situação proposta no inquérito, relacionada à implantação do SI-PNI, permitiu verificar as maiores fragilidades desse 
Tabela 1 - Coberturas vacinais proporcionais por tipo de vacina em menores de 1 ano de idade e respectivas metas (\%), Roraima, 2013-2017

\begin{tabular}{lcccccc}
\hline Vacina & 2013 & 2014 & 2015 & 2016 & 2017 & Meta de cobertura \\
\hline Bacilo de Calmette-Guérin & 126,6 & 146,1 & 112,7 & 107,3 & 113,6 & 90,0 \\
Rotavírus humano & 70,4 & 82,4 & 94,2 & 87,8 & 90,9 & 90,0 \\
Pneumocócica 10-valente & 80,3 & 80,7 & 89,7 & 101,7 & 97,9 & 95,0 \\
Meningocócica conjugada C & 81,5 & 81,1 & 95,6 & 99,1 & 89,5 & 95,0 \\
Pentavalente & 80,4 & 79,6 & 96,5 & 92,4 & 92,6 & 95,0 \\
Poliomielite & 84,3 & 84,7 & 106,6 & 89,2 & 88,9 & 95,0 \\
Febre amarela & 79,8 & 82,8 & 92,3 & 89,1 & 95,2 & 100,0 \\
\hline
\end{tabular}

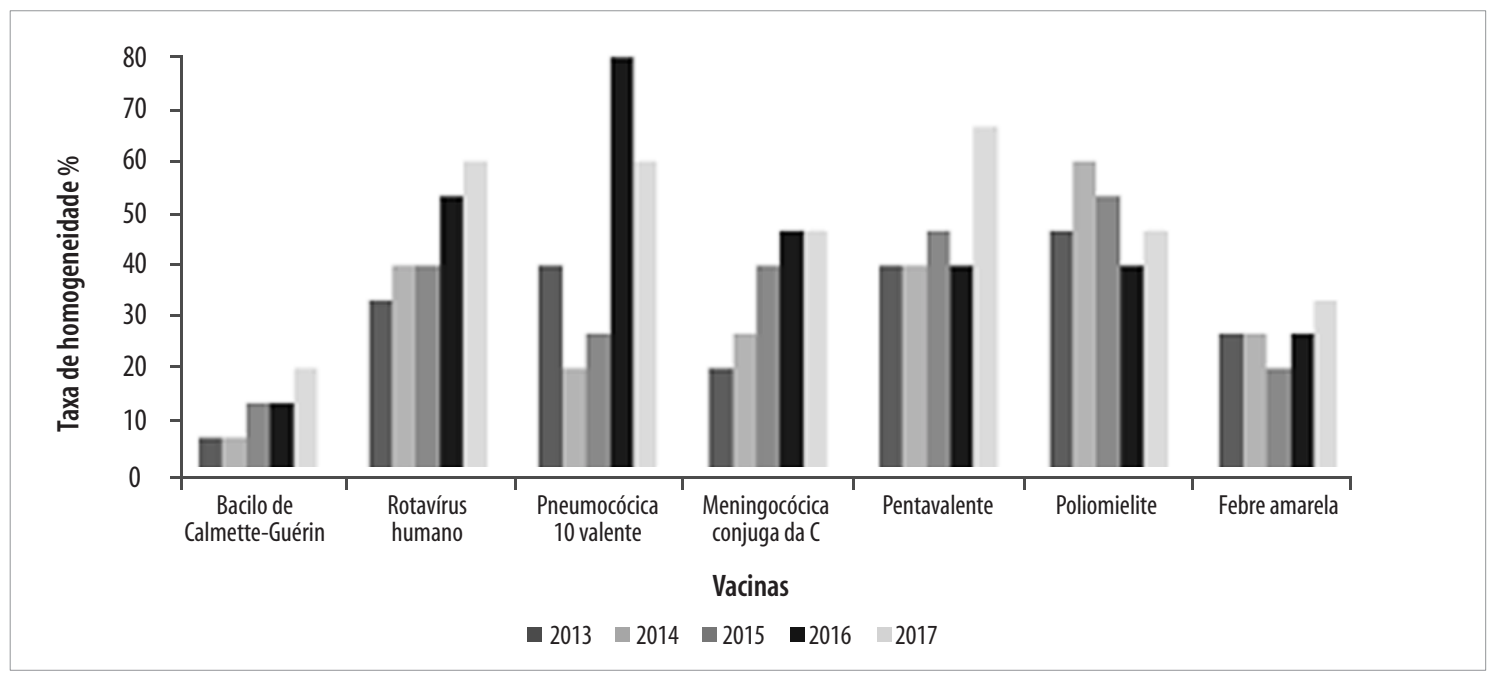

Nota: a linha indica o nível de taxa de homogeneidade de 70\%.

Figura 1 - Homogeneidade de coberturas vacinais, por tipo de vacina, Roraima, 2013-2017

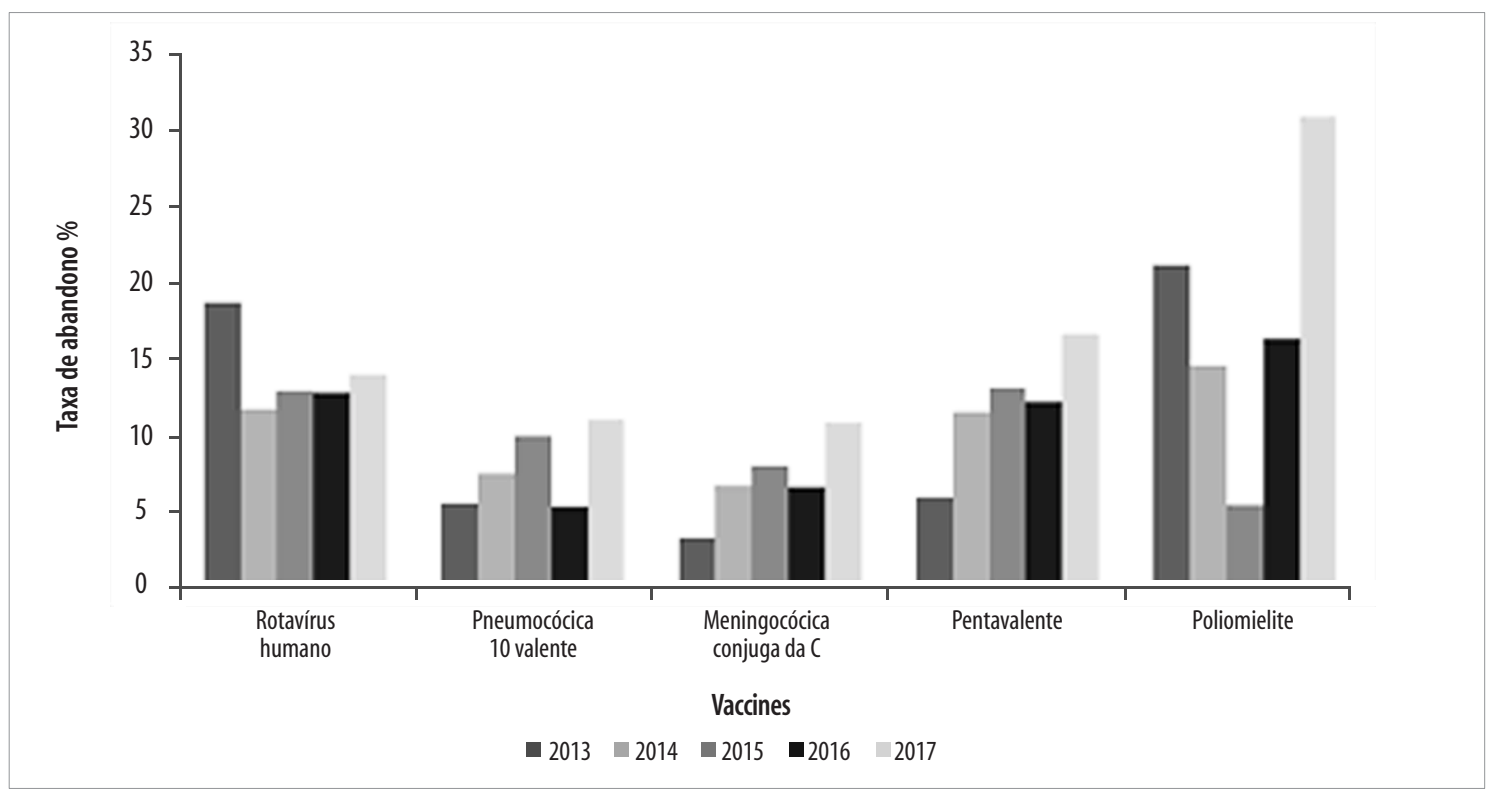

Figura 2 - Taxa de abandono da vacinação de crianças menores de 1 ano, Roraima, 2013-2017 
Tabela 2 - Frequência (\%) das percepções dos profissionais de saúde e gestores $(\mathrm{N}=100)$ quanto às barreiras para 0 alcance de elevadas coberturas vacinais, Roraima, 2017

\begin{tabular}{lcccc}
\hline Fragilidade no processo de implantação do SI-PNI ${ }^{a}$ & Pouco frágil & Frágil & Sem fragilidade & Muito frágil \\
\hline Falta de qualificação dos profissionais & 25,0 & 40,0 & 10,0 & 25,0 \\
Computadores e sua instalação & 19,0 & 24,0 & 24,0 & 33,0 \\
Dificuldade de acesso à internet & 12,0 & 23,0 & 9,0 & 56,0 \\
Sobrecarga de trabalho & 15,0 & 26,0 & 11,0 & 48,0 \\
Demora no atendimento & 28,0 & 22,0 & 10,0 & 40,0 \\
Quantidade insuficiente de profissionais & 17,0 & 19,0 & 10,0 & 54,0 \\
\hline Dificuldade no processo de adesão ao programa de imunizações & Nenhuma & Pouca & Nem pouca, nem & Muita \\
Dificuldade de acesso ao serviço/extensa área rural & 16,0 & 37,0 & 25,0 & 22,0 \\
Hesitação à vacinação & 8,0 & 50,0 & 22,0 & 20,0 \\
Falta de investimento no programa & 15,0 & 26,0 & 27,0 & 32,0 \\
Falta de prioridade para o programa & 18,0 & 24,0 & 24,0 & 34,0 \\
Falta de recursos financeiros & 18,0 & 26,0 & 24,0 & 32,0 \\
\hline
\end{tabular}

a) SI-PNI:Sistema de Informações do Programa Nacional de Imunizações.

processo, segundo eles: dificuldades de acesso à internet $(56 \%)$, quantidade insuficiente de profissionais (54\%) e sobrecarga de trabalho (48\%).

Os valores apontados para os fatores responsáveis pela dificuldade de adesão da população ao programa de imunizações mostraram-se próximos, entre as variáveis (Tabela 2), embora cada uma delas se destacasse pela diversidade das opiniões: por exemplo, a 'falta de investimento no programa' foi percebida como uma influência muito intensa para $32 \%$ dos participantes e sem importância (nenhuma) para $15 \%$.

A variável apresentada como dificultadora de adesão ao programa de imunizações com maior diferença de opiniões entre os participantes - desde os que perceberam sua influência mais intensa àqueles para os quais ela foi menos intensa - foi a 'hesitação à vacinação': enquanto $50 \%$ dos participantes da pesquisa consideraram-na de pouca influência, para $22 \%$ essa influência foi muita, e para $8 \%$, a variável não teve nenhuma influência na adesão ao programa de vacinações (Tabela 2 ).

\section{Discussão}

Os resultados desta pesquisa indicaram, de modo geral, coberturas vacinais inferiores às metas estabelecidas pelo Ministério da Saúde; outras coberturas, muito acima dessas metas; e oscilações, a ponto de um ano apresentar excelente cobertura vacinal $\mathrm{e} o$ ano seguinte, uma drástica queda proporcional.
Uma limitação do estudo é inerente ao uso de dados secundários, sujeitos a possíveis erros de digitação, incompletude ou pendências de inserção nos sistemas de informações de imunizações - ou seja, a possibilidade de registro de novos dados após a realização desta pesquisa.

Apenas um a cada 2,3 questionários, enviados aos profissionais registrados como atuantes no programa de imunizações, foi respondido. Considerando-se a alta rotatividade dos gestores/profissionais de saúde e a contratação de novos vacinadores, é possível que os endereços eletrônicos cadastrados nos sistemas não estivessem atualizados. Apesar da possibilidade de a baixa proporção de resposta ter influenciado a capacidade do estudo em representar a população-alvo, os resultados deste trabalho corroboram os da literatura nacional e internacional: as barreiras de acesso a serviço influenciam fortemente a adesão da população aos programas de prevenção de doenças. ${ }^{16}$ Contudo, se a hesitação à vacinação é um dos fatores responsáveis pela queda das coberturas vacinais no Brasil, sua influência é baixa na adesão ao programa em Roraima. ${ }^{17}$

A cobertura vacinal da BCG no estado de Roraima manteve-se alta, alcançando mais de $100 \%$ durante todo o período estudado. Este achado vai de encontro aos de estados como Pará, Maranhão e Bahia, onde se observou um decréscimo no número de vacinados com esse imunobiológico. A heterogeneidade das coberturas vacinais nos estados brasileiros 
pode ser explicada por nascimentos fora de local de residência da mãe. ${ }^{18}$ Nesse sentido, é possível que a estimativa da população de crianças residentes em Roraima esteja subestimada, devido ao alto número de migrantes e seu possível impacto nos achados de cobertura vacinal do estudo.

Em 2015, as coberturas vacinais da poliomielite foram elevadas e no ano seguinte, contrariamente, muito inferiores às metas estabelecidas. Tais oscilações podem refletir erros nos registros, não inserção de dados por dificuldades logísticas e/ou, ainda, subnumeração ou subenumeração populacional. ${ }^{19}$ Não obstante, a existência de países onde a doença é endêmica, como Afeganistão, Paquistão e Nigéria, ${ }^{20}$ indicam que as baixas coberturas vacinais observadas representam um risco de reemergência da poliomielite no Brasil. Portanto, esforços devem ser realizados para 0 alcance das metas de vacinação nos próximos anos.

Neste estudo, observou-se um considerável aumento das taxas de abandono da vacinação contra poliomielite e rotavírus humano. Quanto maior for a credibilidade no programa de vacinações, maiores tenderão a ser as coberturas vacinais e, por conseguinte, a taxa de abandono diminuirá. ${ }^{21} 0$ acesso da população ao serviço de vacinação também interfere nas taxas de abandono, a exemplo de barreiras geográficas - e.g. a extensa área rural e indígena - e barreiras organizacionais, ambas reconhecidas como condições dificultadoras para a implantação do novo sistema de informações, haja vista a dificuldade de acesso à internet em determinadas localidades, e regiões do país inclusive. ${ }^{22}$

Embora a hesitação à vacinação não seja considerada uma das maiores responsáveis pela não vacinação em Roraima, esse fator é apontado como um dos maiores responsáveis pela queda das coberturas vacinais no país. Trata-se de uma constatação igualmente comum em regiões com acesso garantido às vacinas. É o caso da Europa, onde não há barreiras de vacinação relacionadas a infraestrutura e sim barreiras socioculturais, como crenças religiosas ou filosóficas questionadoras ou mesmo contrárias à vacinação. ${ }^{23}$

O desabastecimento de imunobiológicos, atribuído a dificuldades na entrega de vacinas internacionais e/ou à capacidade de produção dos laboratórios, pode ser responsabilizado pela queda das coberturas vacinais no Brasil. ${ }^{23} \mathrm{~A}$ despeito desses problemas, em Roraima, não se identificou associação da falta de imunobiológicos com a redução das coberturas vacinais no estado.

Analisar as coberturas vacinais permite verificar 0 desempenho do programa de imunizações de Roraima e conhecer os grupos suscetíveis a adquirir doenças imunopreveníveis. Os resultados desta pesquisa revelaram heterogeneidade nas coberturas vacinais entre os municípios de Roraima, e altas taxas de abandono, principalmente da vacinação contra o rotavírus humano e a poliomielite. Ficou evidenciado, ademais, que barreiras de acesso à vacinação influenciam no alcance de coberturas vacinais elevadas.

\section{Contribuições das autoras}

Fonseca KR e Buenafuente SMF contribuíram com a concepção, delineamento do estudo e interpretação dos dados. Fonseca KR contribuiu com a redação do manuscrito e Buenafuente SMF realizou a revisão crítica do conteúdo intelectual. Ambas as autoras aprovaram a versão final e são responsáveis por todos os aspectos do trabalho, incluindo a garantia de sua precisão e integridade.

\section{Referências}

1. Méndez MCR, Barros AJD, Wong KLM, Johnson HL, Pariyo G, Wehrmeister FC, et al. Missed opportunities in full immunization coverage: findings from low- and lower-middle-income countries. Global Health Action [Internet]. 2016 May [cited 2020 Dec 29];9(1):30963. Available from: https://doi. org/10.3402/gha.v9.30963

2. Hochman G. Vacinação, varíola e uma cultura da imunização no Brasil. Ciênc Saúde Coletiva
[Internet]. 2011 fev [citado $2020 \mathrm{dez} 29$ ];16(2):37586. Disponível em: https://doi.org/10.1590/S141381232011000200002

3. Domingues CMAS, Woycicki JR, Rezende KS, Henriques CMP. Programa Nacional de Imunização: a política de introdução de novas vacinas. Rev Eletr Gestão Saúde [Internet]. 2015 out [citado 2020 dez 29];6(supl 4):3250-74. Disponível em: https:// periodicos.unb.br/index.php/rgs/article/view/3331 
4. Ministério da Saúde (BR). Secretaria de Vigilância em Saúde. Departamento de Vigilância Epidemiológica. Programa Nacional de Imunizações (PNI): 40 anos [Internet]. Brasília: Ministério da Saúde; 2013 [citado $2020 \mathrm{dez} 29] .236$ p. Disponível em: https:// bvsms.saude.gov.br/bvs/publicacoes/programa_ nacional_imunizacoes_pni40.pdf

5. Ministério da Saúde (BR). Secretaria de Vigilância em Saúde. Departamento de Vigilância das doenças transmissíveis. Manual de normas e procedimentos para vacinação [Internet]. Brasília: Ministério da Saúde; 2014 [citado 2020 dez 29]. 176 p. Disponível em: http://bvsms.saude.gov.br/bvs/publicacoes/ manual_procedimentos_vacinacao.pdf

6. Silva ABS, Araújo ACM, Santos MCS, Andrade MS, Mendonça RM. Indicadores de Cobertura Vacinal para classificação de risco de doenças imunopreveníveis. Rev Bras Promoç Saúde [Internet], 2019 ago; 32(1):1-11. Disponível em: https://periodicos.unifor. br/RBPS/article/view/9285

7. Domingues CMAS, Teixeira AMS. Coberturas vacinais e doença imunopreveníveis no Brasil no período 1982-2012: avanços e desafios do programa nacional de imunizações. Epidemiol Serv Saúde [Internet]. 2013 jan-mar [citado $2020 \mathrm{dez} 29$ ];22(1):9-27. Disponível em: http://dx.doi.org/10.5123/S167949742013000100002

8. Sato APS. National Immunization Program: computerized system as a tool for new challenges. Rev Saúde Pública [Internet]. 2015 Jul [cited 2020 Dec 29]; 49:39:1-5. Available from: https://doi. org/10.1590/S0034-8910.2015049005925

9. Kagoné M, Yé M, Nébié E, Sie A, Schoeps A, Becher $\mathrm{H}$, et al. Vaccination coverage and factors associated with adherence to the vaccination schedule in young children of a rural area in Burkina Faso. Global Health Action [Internet]. 2017 Nov [cited 2020 Dec 29];10(1):1399749. Available from: https://doi.org/1 $0.1080 / 16549716.2017 .1399749$

10. Tanaka OY, Tamaki EM. O papel da avaliação nos serviços de saúde para tomada de decisão. Ciênc Saúde Coletiva [Internet]. 2012 [citado 2020 $\operatorname{dez} 29] ; 17(4): 821-8$. Available from: https://doi. org/10.1590/\$1413-81232012000400002

11. Barbosa LA, Sales AFG, Souza ILL. Reflexos da imigração venezuelana na assistência em saúde no maior hospital de Roraima: análise qualitativa. Saúde Soc [Internet]. 2020 jun [citado $2020 \mathrm{dez}$ 29];29(2):1-11. Disponível em: http://dx.doi. org/10.1590/s0104-12902020190730
12. Instituto Brasileiro de Geografia e Estatística - IBGE. População residente estimativas para o TCU Roraima, 2017 [Internet]. Rio de Janeiro: IBGE; 2017 [citado 2020 dez 29]. Disponível em: http://tabnet.datasus. gov.br/cgi/tabcgi.exe?ibge/cnv/poptrr.def

13. Ministério da Saúde (BR). Nascidos Vivos - Roraima, 2013 - 2017 [Internet]. Brasília: Ministério da Saúde; 2017 [citado 2020 dez 29]. Disponível em: http://tabnet.datasus.gov.br/cgi/tabcgi.exe?sinasc/cnv/ nvrr.def

14. Ministério da Saúde (BR). Secretaria de Vigilância em Saúde. Coberturas vacinais no Brasil: período: 2010 - 2014 [Internet]. Brasília: Ministério da Saúde; 2015 [citado $2020 \mathrm{dez} 29$ ]. Disponível em: https:// antigo.saude.gov.br/images/pdf/2017/agosto/17/ AACOBERTURAS-VACINAIS-NO-BRASIL---2010-2014. pdf

15. Ferreira AV, Oliveira CF, Guimarães EAA, Cavalcante $\mathrm{RB}$, Moraes JT, Oliveira VC. Acesso às salas de vacina na Atenção Primária à Saúde. Rev Eletr Enf [Internet] . 2017 ago [citado $2020 \mathrm{dez} 29] ; 19(1): 1-11$. Disponível em: https://doi.org/10.5216/ree.v19.42468

16. Figueiredo A, Johnston LG, Smith DMD, Agarval S, Larson H, Jones NS. Forecasted trends in vaccination coverage and correlations with socioeconomic factors: a global time-series analysis over 30 years. Lancet Glob Health [Internet]. 2016 Oct [cited 2020 Dec 29];4(10):726-35. Available from: https://doi. org/10.1016/S2214-109X(16)30167-X

17. Duarte DC, Oliveira VC, Guimarães EAA, Viegas SMF Vaccination access in Primary Care from the user's perspective: senses and feelings about healthcare services. Escola Anna Nery [Internet]. 2018 Dec [cited 2020 Dec 29];23(1):1-8. Available from: https://doi.org/10.1590/2177-9465-ean-2018-0250

18. Arroyo LH, Ramos ACV, Yamamura M, Weiller TH, Crispim JA, Ramos DC, et al. Áreas com queda da cobertura vacinal para BCG, poliomielite e tríplice viral no Brasil (2006-2016): mapas da heterogeneidade regional. Cad Saúde Pública [Internet]. 2020 [citado $2020 \mathrm{dez} 29$ ];36(4). Disponível em: https://doi.org/10.1590/0102311X00015619

19. Teixeira AMS, Rocha CMV. Vigilância das coberturas de vacinação: uma metodologia para detecção e intervenção em situações de risco. Epidemiol Serv Saúde [Internet]. 2010 jul-set [citado $2020 \mathrm{dez}$ 29];19(3):217-26. Disponível em: http://dx.doi. org/10.5123/S1679-49742010000300004 
20. Ministério da Saúde (BR). Informe técnico: campanha nacional de vacinação contra a Poliomielite e contra o Sarampo [Internet]. Brasília: Ministério da Saúde; 2018 [citado 2020 dez 29]. Disponível em: https://www.cevs.rs.gov. br/upload/arquivos/201807/31163732-informetecnico-campanha-nacional-de-vacinacao-contra-apoliomielite-e-o-sarampo-2018.pdf

21. Braz MR, Domingues CMAS, Teixeira AMS, Luna EJA. Classificação de risco de transmissão de doenças imunopreveníveis a partir de indicadores de coberturas vacinais nos municípios brasileiros. Epidemiol Serv Saúde [Internet]. 2016 out-dez [citado 2020 dez 29];25(4):745-54. Disponível em: https://doi.org/10.5123/s1679-49742016000400008

22. Silva BS, Coelho HV, Cavalcante RB, Oliveira VC, Guimarães EAA. Evaluation study of the National Immunization Program Information System. Rev Bras Enfermagem [Internet]. 2018 Nov [cited 2020 Dec 29];71(1):615-24. Available from: http://dx.doi. org/10.1590/0034-7167-2017-0601

23. Sato APS. What is the importance of vaccine hesitancy in the drop of vaccination coverage in Brazil? Rev Saúde Pública [Internet]. 2018 Nov [cited 2020 Dec 29];52(1):1-9. Available from: https://doi. org/10.11606/s1518-8787.2018052001199

\section{Resumen}

Objetivo: Analizar la cobertura de vacunación de los menores de un año en Roraima, Brasil, entre 2013 y 2017 , y exponer las percepciones de los profesionales, sobre las barreras que influyen en el logro de una mejor cobertura, en 2017. Métodos: Estudio descriptivo que analizó las coberturas para el bacilo de Calmette y Guérin (BCG), rotavirus, poliomielitis, fiebre amarilla, pentavalente, meningococo $C$ conjugado y neumocócica 10 valente, basado en datos de los sistemas de información de inmunizaciones. Las barreras percibidas por los profesionales se midieron en un cuestionario basado en la escala Likert, por entrevista o autoaplicación. Resultados: La mayor cobertura fue de BCG $(146,1 \%)$ en 2014; y la más baja, de rotavirus $(70,4 \%)$ en 2013. La principal barrera (56/100) identificada por los profesionales que trabajan en el programa (100 participantes) para una mejor cobertura fue la dificultad de acceso a la internet. Conclusión: La baja cobertura de vacunación refleja la influencia de las barreras para acceder a la vacunación.

Palabras clave: Cobertura de Vacunaciónl; Programas de Inmunización; Vacunación; Accesibilidad a los Servicios de Salud; Epidemiología descriptiva.

Recebido em 24/04/2020

Aprovado em 09/11/2020 